\title{
Cervical Lymphadenopathy in a Nonagenarian Woman: What to Think?
}

\author{
Vanessa Meireles Chaves, Fernando Miguel Miranda Nogueira, Gilberto Pires da Rosa, Sofia Tavares, \\ Inês Ferreira, Ana Oliveira Monteiro, Ester Maria Morgado Ferreira \\ Department of Internal Medicine, Centro Hospitalar Universitário de S. João, Porto, Portugal
}

Doi: 10.12890/2019_001336 - European Journal of Case Reports in Internal Medicine - ๔ EFIM 2019

Received: $16 / 10 / 2019$

Accepted: 23/10/2019

Published: 03/12/2019

How to cite this article: Meireles Chaves V, Miranda Nogueira FM, Pires da Rosa G, Tavares S, Ferreira I, Monteiro AO, Morgado Ferreira EM. Cervical lymphadenopathy in a nonagenarian woman: what to think? EJCRIM 2019;6: doi:10.12890/2019_001336.

Conflicts of Interests: The Authors declare that there are no competing interest.

This article is licensed under a Commons Attribution Non-Commercial 4.0 License

\section{ABSTRACT}

Tuberculosis remains a worldwide public health problem. Cervical tuberculous lymphadenitis (TBL) or scrofula is the most common form of extrapulmonary tuberculosis, affecting the cervical lymph nodes. We report the case of a 93-year-old woman presenting with cervical adenopathies with 3 months duration. Fine needle aspiration (FNA) biopsy yielded a noncaseous granulomatous process, but was negative for Mycobacterium tuberculosis (MT). As the adenopathies had grown, an excisional biopsy was performed. An extensive study of infectious aetiologies was performed, including for MT, with a negative outcome. Owing to the persistence of cervical lymphadenitis with caseous granulomas, a diagnosis of TBL was strongly suspected and presumptive treatment was initiated. Afterwards, diagnostic confirmation was obtained by isolation of MT in the lymph node culture. The patient presented a favourable clinical outcome. This case highlights that a high index of suspicion is essential for the diagnosis of TBL, especially in the elderly, and emphasizes the importance of pursuing diagnostic confirmation, in which FNA and excisional biopsy plays a key role.

\section{LEARNING POINTS}

- The diagnosis of tuberculous lymphadenitis should be considered in all patients presenting with cervical adenopathies, especially in endemic areas, irrespective of the patient's age.

- A high index of suspicion is essential for the diagnosis due to the wide range of conditions that can mimic the disease.

- Fine needle aspiration biopsy is appropriate as an initial diagnostic approach, with excisional biopsy recommended when the former is not diagnostic.

\section{KEYWORDS}

Cervical lymphadenopathy, cervical tuberculous lymphadenitis, fine needle aspiration biopsy, excisional biopsy, differential diagnosis

\section{CASE PRESENTATION}

A 93-year-old Caucasian woman was referred to the Internal Medicine Consultation due to cervical adenopathies with 3 months duration. With respect to past medical history, 4 years earlier she had been diagnosed with cutaneous sarcomatoid carcinoma of the face and submitted to surgical excision. The patient denied having fever, asthenia, fatigue, weight loss or night sweats; furthermore, there were no respiratory symptoms. She denied having a previous history of tuberculosis (TB) and recent contact with people with known or suspected TB. There was no epidemiological context or contact with animals. On physical examination, an enlarged, mobile and painful right cervical lymph node was evident; the remainder of the examination was unremarkable. 
Methods and Procedures

Cervico-thoraco-abdominal-pelvic computed tomography (CT) showed a right cervical mass (Fig. 1) with multiple adenopathies and some fibrotic lesions in the upper right lobe; there were no other adenopathies nor evidence of neoplastic disease. Fine needle aspiration (FNA) biopsy of the cervical node was performed and yielded a noncaseous granulomatous process. There was no evidence of Reed-Sternberg cells or neoplastic cells; Ziehl-Neelsen staining was negative, as well as polymerase chain reaction (PCR) assessment for Mycobacterium tuberculosis (MT) and culture. The patient was kept under close surveillance. In the meantime, an IgG lambda monoclonal gammopathy was diagnosed; renal function and calcium metabolism were normal, there was no anaemia or elevated urinary free light chains and beta- 2 microglobulin was normal. Peripheral blood immunophenotyping demonstrated a polyclonal B cell population. In addition, serology tests for syphilis, human immunodeficiency virus (HIV), hepatitis B virus and hepatitis C virus were negative, and QuantiFERON testing was positive. Two months later, the adenopathies had grown and there was cutaneous fistulization with suppurative lymphadenitis (Figs. 2A and 2B).

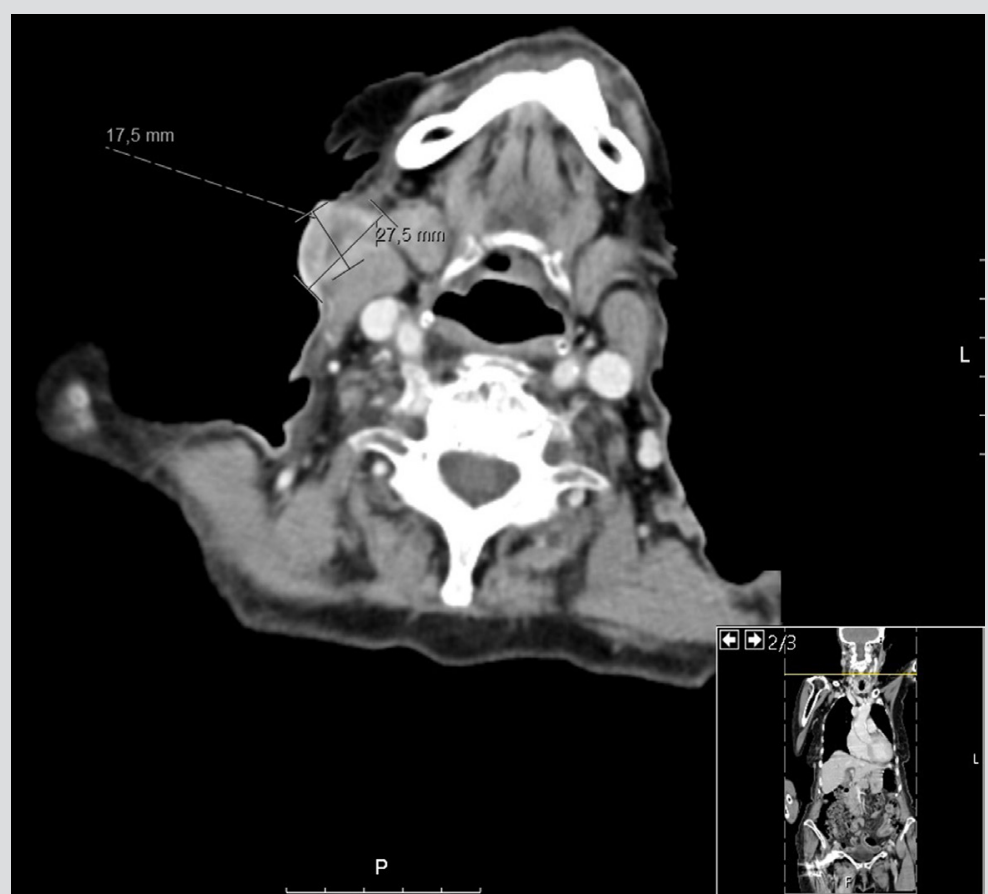

Figure 1. Cervical computed tomography scan in axial view displaying a right cervical mass of $27.5 \times 17.5 \mathrm{~mm}$. P denotes posterior, $L$ denotes left

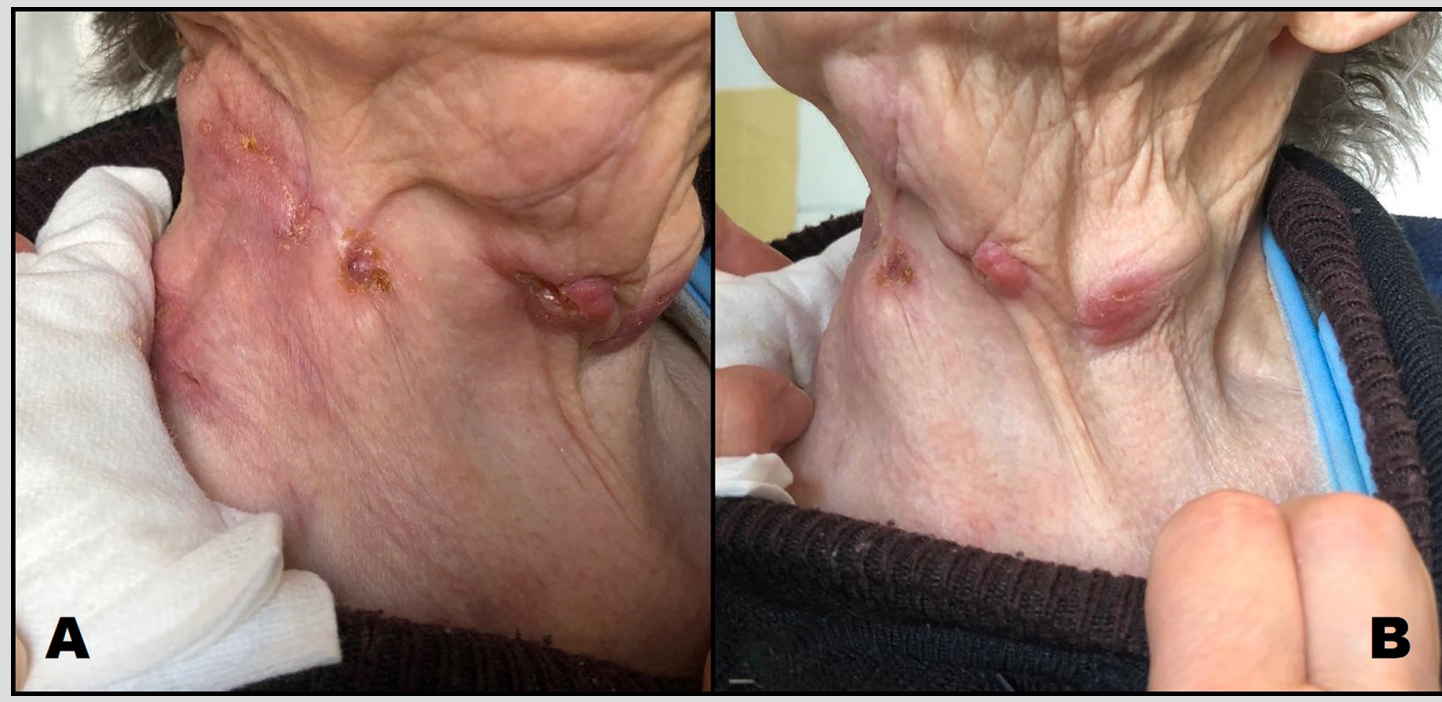

Figure 2. Right (A) and left (B) cervical adenopathies with cutaneous fistulization and suppuration 
Microbiologic examination of a suppuration swab was positive for Staphylococcus aureus and the patient was treated with cefuroxime, displaying only slight improvement. An excisional biopsy was programmed and histological examination revealed granulomas with caseous necrosis and suppuration.

PCR assessment of the samples was negative for MT, Nocardia, Mycobacterium avium complex, Toxoplasma gondii, Aspergillus fumigatus, Bartonella spp., Treponema pallidum and Leishmania infantum. Considering the presence of persistent cervical lymphadenopathy with caseous granulomas, a positive QuantiFERON test, and the exclusion of other potential causes, the hypothesis of cervical tuberculous lymphadenitis was strongly suspected, even without a positive PCR test for MT. The patient started presumptive treatment with isoniazid, rifampicin, pyrazinamide and ethambutol. Afterwards, diagnostic confirmation was obtained by isolation of MT in one of the lymph node cultures. Antimicrobial susceptibility testing did not demonstrate resistances. The patient presented a favourable clinical outcome with gradual reduction in the size of the adenopathies and there were no side effects attributable to the antituberculous drugs.

\section{DISCUSSION}

Tuberculosis (TB) remains a worldwide public health problem with a high incidence in the north of Portugal. In the city of Porto, in 2014, the reported incidence rate was 46.3 cases per 100,000 inhabitants. The higher incidence of TB in European urban areas has been associated with the phenomenon of immigration from high-TB-incidence countries and with a subgroup of the population with risk factors, namely people who are HIV-infected, drug users, high alcohol consumers, prisoners and the homeless ${ }^{[1]}$. Several studies have reported that, overall, $75 \%$ of TB cases show exclusive pulmonary involvement (PTB), 19\% have only extrapulmonary disease (EPTB) and 6\% present with mixed disease. While PTB patients have, in general, more severe forms of disease compared with EPTB cases and a higher risk of direct transmissibility, EPTB also represents a public health problem due to the challenges in diagnosis, that can delay treatment and predispose to progression of disease, leading to significant clinical sequelae ${ }^{[2]}$.

The most common site of EPTB is the lymph nodes, a condition termed cervical tuberculous lymphadenitis (TBL) or scrofula. It is considered to be the local manifestation of the systemic disease, typically caused by reactivation of latent Mycobacteria found within the lymph nodes that were seeded during primary infection. In the United States, TBL accounts for $30 \%$ to $50 \%$ of EPTB cases ${ }^{[3]}$, with cervical lymph nodes being most commonly affected ${ }^{[3,4]}$. TBL has been identified most frequently in children and, in recent years, it has become common in young adults (20 to 40 years old), with a female predilection ${ }^{[2,3]}$. In our case, the diagnosis of TBL was established in a 93-year-old woman, which is probably related to the reactivation of infection acquired in the remote past.

Clinical presentation is usually indolent, consisting of non-tender slow enlargement of cervical lymph nodes, often unilateral, involving one or more nodes of the anterior and posterior cervical chain. Constitutional symptoms are often absent in immunocompetent individuals ${ }^{[3]}$, as was seen in the case we report.

Diagnosis of TBL is challenging and can be overlooked due to the wide range of infectious and non-infectious (neoplastic and systemic) conditions that share identical clinical presentation. Imaging techniques such as ultrasound, CT or magnetic resonance imaging of the neck are useful for initial evaluation of lymphadenopathy and can identify the lymph node group most suitable for biopsy, as well as assist in the biopsy $^{[5]}$. The "gold standard" for diagnosis remains the culture, although it may take up to 3 to 4 weeks to yield results, which represents a major delay in establishing the diagnosis when malignant processes need to be excluded. A definitive diagnosis of TBL can also be made by PCR demonstration of MT in an affected lymph node. Histologic features, such as an epithelioid granulomatous reaction, accompanied or not by caseation and necrosis, support the diagnosis ${ }^{[3]}$. FNA biopsy is a safe and minimally invasive procedure that provides a good clinical specimen suitable for laboratory work-up; therefore, it is appropriate as an initial approach. On the other hand, excisional biopsy for histopathologic and microbiological analysis has the highest diagnostic yield, and should be pursued when FNA biopsy is inconclusive ${ }^{[3,4]}$.

In our case, considering the patient's age and previous history of cancer, the first diagnostic hypothesis was a neoplastic condition, which became less probable after the FNA biopsy results. Considering the histological features of caseous granulomas, several infectious conditions (bacterial, viral, parasitic and fungal) were investigated and excluded. Regarding inflammatory diseases, sarcoidosis could also have a similar presentation, but was unlikely given the age of the patient and the absence of other imaging and analytical supportive findings. Due to the high index of suspicion of TBL in an endemic area, treatment was started presumptively, without microbiologic confirmation. The persistence in searching for MT ultimately led to diagnostic confirmation by culture. As we report in this case, diagnosis of TB in the elderly can be difficult, due to non-specific clinical features. Nevertheless, due to the high index of suspicion, diagnosis was established in a timely manner and appropriate treatment initiated, with an excellent recovery.

In conclusion, we report a case of cervical tuberculous lymphadenitis with suppuration in a nonagenarian woman. With this report, we would like to increase the awareness of clinicians with respect to this entity, which should be suspected in all patients presenting with cervical adenopathies, mainly in endemic areas, irrespective of the patient's age. 


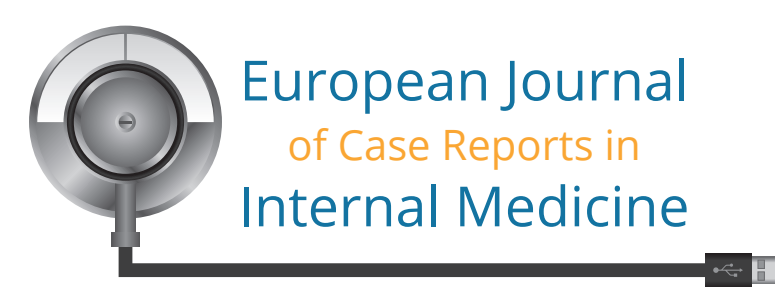

We also emphasize that a high index of suspicion is of utmost importance for a timely diagnosis and treatment initiation. Moreover, due to the possible side effects of antituberculous treatment, it is important to weigh the risk/benefits of presumptive treatment initiation, especially in the elderly. Finally, our case shows that, even though techniques for detection of MT are well developed, the isolation and identification of MT in the lymph nodes can be difficult. Persistence in searching for this bacterium is crucial for diagnostic confirmation. In this regard, FNA biopsy and excisional biopsy have a key role in the diagnosis.

\section{REFERENCES}

1. Rito T, Matos C, Carvalho C, Machado H, Rodrigues G, Oliveira O, et al. A complex scenario of tuberculosis transmission is revealed through genetic and epidemiological surveys in Porto. BMC Infect Dis 2018;18:53.

2. Sotgiu G, Falzon D, Hollo V, Ködmön C, Lefebvre N, Dadu A, et al. Determinants of site of tuberculosis disease: an analysis of European surveillance data from 2003 to 2014. PLoS One 2017;12:e0186499.

3. Cataño JC, Robledo J. Tuberculous lymphadenitis and parotitis. Microbiol Spectr 2016;4(6):TNMI7-0008-2016.

4. Moualed D, Robinson M, Qureishi A, Gurr P. Cervical tuberculous lymphadenitis: diagnosis and demographics, a five-year case series in the UK. Ann R Coll Surg Engl 2018;100:392-396

5. Gambhir S, Ravina M, Rangan K, Dixit M, Barai S, Bomanji J; International Atomic Energy Agency Extra-pulmonary TB Consortium. Imaging in extrapulmonary tuberculosis. Int J Infect Dis 2017;56:237-247. 